Fecha de recepción: diciembre 2017

Fecha de aceptación: marzo 2018

Versión final: julio 2019

\section{El “diseño" como valor y la conformación de un universo de creencia ${ }^{1}$}

Paula Miguel $^{*}$

Resumen: Este trabajo analiza el proceso de valorización, legitimación y creciente autonomía que adquiere el diseño de indumentaria a partir del año 2000 en Buenos Aires, centrándose en diseñadores profesionales-empresarios. Usando como disparador la preocupación por los mundos de la "alta cultura", la producción cultural, estética y de valor simbólico en la obra de Pierre Bourdieu, se retoman sus ideas a propósito de los "universos de creencia" para articular la presencia de diferentes actores con lógicas particulares en un espacio relacional compartido por productores, productos, intermediarios y público, donde se construyen, reproducen y renuevan gustos y preferencias, donde se negocian posicionamientos y se plantean jerarquías.

Palabras clave: Diseño - Indumentaria - Producción simbólica - Emprendedorismo.

[Resúmenes en inglés y portugués en la página 142]

${ }^{(*)}$ Socióloga, especializada en Sociología de la cultura y análisis cultural y Doctora en Ciencias Sociales por la Universidad de Buenos Aires. Es Profesora Titular de Sociología en la Facultad de Arquitectura, Diseño y Urbanismo e investigadora y docente en la Facultad de Ciencias Sociales de esa universidad. Su trabajo de investigación se enfoca en el análisis de las industrias creativas, la producción simbólica, el emprendedorismo estético-cultural en los sectores medios y las dimensiones culturales de la economía. paulamiguel@gmail.com

\title{
Introducción
}

¿Qué elementos presentes en la obra de Pierre Bourdieu pueden contribuir al análisis del diseño de indumentaria local? ¿Cómo logró definirse un espacio específico de producción de este tipo de diseño? ¿De qué manera se construyó un conocimiento tácito sobre el diseño a la par que se desarrolla en tanto espacio productivo? La preocupación por los mundos de la "alta cultura" y por la producción cultural ha ocupado una parte significativa de la obra de Pierre Bourdieu. Las líneas de abordaje que propone para el análisis de la producción intelectual y artística (Bourdieu, 1988, 1990, 1991, 1995, 2000, 2003) son disparadores teóricos para pensar de manera dinámica y compleja la producción cultural. En el 
caso de este trabajo, utilizadas como herramientas, de manera heurística, resultan productivas para comprender y explicar los procesos vinculados a las dinámicas de producción del diseño (de indumentaria). Procesos que van más allá de la consolidación de espacios académicos, curriculares o profesionales y que presentan particularidades: pese a estar inscriptos en un mercado global, mantienen características locales; pese a estar orientados al mercado y al consumo, rigen en parte su producción según la lógica del "talento" o de la "creación", entre otras tensiones que esta perspectiva teórica permite explorar.

La producción material de objetos culturales, estéticos, artísticos en los análisis de Bourdieu, abarca solo una dimensión básica de su producción. Más importante que eso, es la producción simbólica la que hace a la producción del valor de la obra o trabajo. O, lo que es lo mismo, hace a la creencia en el valor del trabajo, entendido como productor y producto. Una creencia que al mismo tiempo es uno de los principios estructurantes del campo en el que se produce ${ }^{2}$. A partir de allí, en esta oportunidad retomaremos las ideas a propósito de los "universos de creencia" que permiten articular la presencia de diferentes actores con lógicas particulares en un espacio relacional compartido por productores, productos, intermediarios y público, donde se construyen, reproducen y renuevan gustos y preferencias, donde se negocian posicionamientos y se plantean jerarquías. Esto apunta a señalar que, en última instancia, cada espacio produce una illusio específica (Bourdieu 1995:337). Esta illusio está en la base del funcionamiento del espacio productivo, encarnada en el habitus específico, y los agentes que participan no son conscientes de todos sus aspectos, sino que más bien forma parte de eso que no se discute, porque hace a la lógica del propio funcionamiento, regulación y reproducción de las prácticas del campo de manera relativamente autónoma. Esto "tiende a generar todas las conductas "razonables" o de "sentido común" posibles dentro de los límites de estas regularidades que están objetivamente ajustadas a la lógica característica de un determinado campo" (Bourdieu, 1991:97). En este esquema, además de ser la condición y la base de esta puesta en juego de relaciones y prácticas, esta illusio también es, en parte, producto de las mismas. Al mismo tiempo, es posible problematizar e interrogar de manera crítica la productividad de estos aportes para el análisis de estos procesos productivos en el contexto local, enfocándonos específicamente en la producción de diseño de indumentaria.

Para ello, este análisis recupera y combina diferentes fuentes, datos y estrategias de abordaje para analizar las prácticas, representaciones y perspectiva de los actores, sus posicionamientos, así como las redes que construyen de manera relacional. Los datos que dan base a este trabajo provienen de una línea de investigación en curso, centrada en el análisis de productores y emprendedores vinculado con el diseño de indumentaria en la ciudad de Buenos Aires $^{3}$. Una serie de entrevistas en profundidad realizadas a partir del año 2002 abarca, entre otras dimensiones, aspectos en relación con las trayectorias profesionales, las condiciones económicas y su experiencia social y cultural. También se realizaron observaciones en el contexto de las entrevistas, así como en espacios y eventos representativos para el sector, a lo cual se suman los datos provenientes de entrevistas realizadas a otros actores relacionados con el mundo del diseño (periodistas, organizadores de eventos, funcionarios públicos, docentes, etc.). Estos datos se complementan con el material de una base de notas de prensa a partir del análisis de los suplementos de moda y revistas dominicales de los principales diarios argentinos ${ }^{4}$, revistas de moda y artículos recopilados desde 1993 en adelante. 
Aquí interesa el diseño de indumentaria en tanto construcción colectiva localizada, que toma lugar público, que es definida y clasificada de diversas formas, más allá de las definiciones disciplinares, curriculares propias del espacio académico ${ }^{5}$. Se resaltan aquellas dimensiones que tienen que ver con procesos de intermediación simbólica que permiten interpretar el agregado de diseño en los productos como "valor". En ese sentido, si a comienzos de la década de los noventa no se hablaba de diseño de indumentaria y a partir del año 2005 se puede encontrar una categoría nativa como "diseño de autor" instalada en el público más amplio, implica la presencia de procesos de mediación que trabajan activamente en la construcción y difusión de categorías explicativas, que inician y sostienen la creencia en su valor, al tiempo que contribuyen a la valoración de todo el espacio productivo.

Este artículo propone recorrer algunos hitos en el desarrollo del diseño de indumentaria local a partir del año 2000, en tanto producción colectiva del valor del diseño. Como punto de partida, se retoman algunos elementos que tienen que ver con la profesionalización y la institucionalidad académica, que dan la pauta de los clivajes básicos para poder distinguir la especificidad de este tipo de diseño. No obstante, el diseño para ser reconocido como valor debe traspasar las fronteras de la producción restringida hacia públicos más amplios. Allí la prensa tiene un rol importante en la vinculación con públicos amplios y la forma en que el diseño profesional fue puesto en circulación reconociendo y valorando "nombres" de un conjunto llamado "diseño de autor", tal como se lo nominó para introducir la novedad a sus lectores. Por último, se trabaja sobre una manera de pensar el encuentro de productores, mediadores y público en un evento que los nuclea, al tiempo que se discuten las transformaciones de estos espacios en la última década.

\section{La profesionalización y la institución académica como base de la creencia}

Durante los años noventa, los centros urbanos de Argentina se renovaban: junto con la TV por cable, llegaba MTV, nacían los shoppings, que se llenaban rápidamente con productos de marcas "de afuera", los hipermercados se multiplicaban a lo largo y ancho del entramado urbano junto a ofertas de textiles importados, se privatizaban las empresas de servicios públicos y se contrataban servicios de internet que abrían una ventana al mundo. En el marco de esas tendencias, al finalizar la década, ya despuntando los años dos mil, a contrapelo de la crisis económica por la que atravesaba la Argentina (particularmente la industria textil y de la confección) el espacio de producción de moda e indumentaria local se revitalizaba con la aparición de nuevos productos simbólicamente complejos sobre los cuales se destacaba en su difusión el atributo de "diseño" (Saulquin 2006, Guerschman y Vargas 2007,Vargas 2013, Miguel 2013). Estos productos se asocian a un perfil de productores profesionales del diseño, cuya capacidad de innovación y de producción de valor agregado se hará visible y atractiva. Incluso, su conocimiento específico será incorporado paulatinamente por otros actores y sectores de la industria textil y de la confección ${ }^{6}$.

En ese contexto, con el correr de los años, cada vez más y más estudiantes elegían inscribirse en carreras de diseño en la ciudad de Buenos Aires. También comenzaban a presentarse diferentes iniciativas privadas, empresariales, junto a incipientes políticas públicas 
apuntando al desarrollo del diseño, y poco a poco comenzaba a hablarse de diseño de indumentaria en los suplementos de diarios y revistas especializadas. El barrio de Palermo empezaba a ser reconocido como "el barrio del diseño" (Miguel 2015) y Buenos Aires fue nombrada Ciudad de Diseño en 2005 por UNESCO.

Estos elementos permiten pensar que a partir de los años 2000-2001 se abre un proceso de desarrollo y consolidación del diseño de indumentaria como espacio de producción, a la vez que se manifiesta su construcción como valor y es legitimado desde distintos sectores en una producción colectiva de valor simbólico (Bourdieu 2003). Esto tendrá que ver en parte con la profesionalización del diseño y la formación de carreras universitarias, particularmente con la acción de la Carrera de Diseño de Indumentaria y Textil de la Universidad de Buenos Aires. Esta carrera, sus egresados y quienes trabajan en relación con ella tuvieron un papel importante en la creación de espacios institucionales ligados al desarrollo, puesta en circulación y valorización de esta rama del diseño (Miguel 2013). $\mathrm{Al}$ mismo tiempo, la institución académica no agota el proceso. Otros actores, particularmente la prensa especializada, serán también centrales para lograr la valoración del diseño en públicos más amplios, dar cuenta de posiciones y jerarquías, contribuyendo a la “imposición de valor" (Bourdieu y Delsaut, 1975).

La autonomía implica también la capacidad de reproducir en el tiempo ese "algo" específico, independientemente de lo que ocurra con las trayectorias individuales de sus productores. Por eso es que los espacios institucionales, más o menos formales, pueden tomarse como indicadores de dicha autonomía, dado que en la institucionalidad encarna la síntesis de la energía colectiva puesta en juego, en tanto la entidad institucional excede la sumatoria de los interesados en participar de estos espacios.

En un primer momento, en tanto institución académica reconocida a nivel internacional, la Universidad de Buenos Aires promoviendo el desarrollo de la Carrera de Diseño de Indumentaria y Textil, funcionó como motor del desarrollo de la disciplina, acelerando el proceso de institucionalización y autonomía del diseño de indumentaria como espacio de producción específico. Sin embargo, el proceso fue lento, y los primeros años estuvieron signados por una escasa vinculación directa entre la carrera y los sectores de la industria textil y de la confección local. El contexto económico no parecía ser muy auspicioso respecto del porvenir de los jóvenes que empezaban a formarse como diseñadores profesionales, pero sin embargo se inició ese proyecto, promoviendo una serie de relaciones personales, forjadas principalmente en el seno de la carrera, que resultarían de gran importancia para el desarrollo del diseño local.

La profesionalización del diseño en el contexto local y su desarrollo como espacio de producción específico aparece fuertemente correlacionada con la conformación de una currícula académica específica que define la manera en que se forma a los estudiantes dentro de la disciplina, y a su vez dota a la actividad de un espacio institucional. El conjunto de actores vinculados a esas redes, lograron desarrollar con éxito en poco menos de una década un espacio de producción legítimo y reconocido, un mercado, a nivel internacional (Callon et al. 2005).

El boom en la cantidad de estudiantes interesados en ingresar en esta carrera a partir del año 2000 puede ser indicativo de cómo los primeros jóvenes diseñadores egresados de la carrera -sumados al conjunto de agentes implicados en la circulación, distribución y 
valoración de sus productos, en términos tanto simbólicos como económicos-, no sólo consiguieron vender el producto de sus diseños; es decir, que lograron generar exitosamente en distintos niveles el deseo por sus productos, sino que también esta actividad colectiva trasciende el espacio de producción restringida y promueve en otros el deseo de ser diseñador. Esto refiere a la consolidación de un espacio disciplinar específico, cuyas credenciales generan expectativas y, además de ser apreciadas y reconocidas por los propios participantes integrados a la institución, también tienen productividad simbólica y económica por fuera de ella misma. No obstante, los procesos de valorización, legitimación y creciente autonomía que adquiere el diseño de indumentaria desde el año 2000 en adelante, involucran la acción de un conjunto de diferentes actores e instituciones que exceden el desarrollo específico de las carreras vinculadas al diseño de indumentaria en tanto especificidad curricular, que podríamos entender como "campo académico".

\section{Los pares: un grupo de productores en armónica competencia}

El diseño no descansa solamente en la acción de los diseñadores con un título habilitante, de hecho, es un espacio de producción donde las credenciales académicas tienen un peso relativo, ya que deben ser acompañadas de otros atributos como, por ejemplo, vínculos con el campo artístico, obtención de premios, experiencias en el exterior, repercusión en la crítica y prensa especializada, entre otros elementos significativos de la trayectoria profesional (McRobbie 1998).

Entre quienes se destacan en las posiciones más reconocidas entre los diseñadores encontramos un conjunto de diseñadores que, a la luz del entramado ideológico-discursivo de los años noventa, signados por un clima cultural en torno a los valores de la independencia, la capacidad emprendedora y la eficiencia -que luego de los sucesos de diciembre de 2001, que en el marco de la crisis política, social y económica subsiguiente derivó en la autogestión- comparten las bases de un habitus común, en sentido amplio. Dichos elementos se presentan con diferente intensidad y variaciones en cada uno de los miembros, haciendo que el grupo no resulte homogéneo. Cada integrante genera su propia marca, empresa y proyecto conceptual sobre el diseño de indumentaria con su propia estética y particularidades. Se trata de un grupo que, si bien muchos comparten una misma formación, no conforman un todo compacto: es posible rastrear diferentes propuestas, acompañadas por diferentes elecciones en los materiales, diferentes enfoques sobre lo proyectual, sistemas de moldería, entre otros elementos. Cada diseñador va desarrollando su línea de producto al tiempo que genera definiciones discursivas sobre su trabajo, que son retomadas y puestas en tensión por la acción de la prensa especializada.

En base a estas definiciones propias, las de los intermediarios y los resultados de las entrevistas puede observarse una aparente convivencia en armonía dentro del conjunto de pares que conforman las primeras camadas de diseñadores profesionales-empresarios. Aparecen distintas "definiciones" del trabajo de cada uno, diferentes trabajos y concepciones proyectuales a propósito de las formas y el trabajo con los materiales, en el marco de una situación de aparente falta de competencia, en donde cada diseñador tiene su espacio definido y no interfiere, no se superpone, con el de sus pares. 
Si bien una de las principales características que comparten todos los diseñadores de este grupo es el valor que le dan a la superación personal, sea cual fuere el lugar que ocupen en términos de posiciones y jerarquías dentro del espacio productivo, este valor no aparece relacionado con una competencia feroz respecto de los pares, sino como una capacidad de desarrollar el propio potencial y mejorar los propios alcances. A medida que se complejiza el espacio de producción y se incorporan nuevos actores (como, por ejemplo, los graduados más jóvenes que empiezan a trabajar para los primeros diseñadores empresarios y que, al mismo tiempo, comienzan generar nuevas posiciones) la emergencia de nuevas tradiciones conceptuales, estilísticas y estéticas que rompen con las anteriores comienzan a reconfigurar el espacio de posiciones y a marcar líneas de competencia más definidas.

Esta ausencia de marcada competencia y de sana convivencia entre sus integrantes, tal como lo demuestra el desarrollo de emprendimientos conjuntos tales como el de Diseñadores del Bajo $(2000)^{7}$, puede decirse que responde a la necesidad de fortalecerse hacia afuera, mostrando los resultados del aprendizaje de una profesión que no puede basarse puramente en el crédito personal sino, más bien, en el trabajo mancomunado de un grupo de colegas que necesitaban el reconocimiento de la disciplina que validaba su quehacer. Esto contribuye a disminuir la visibilidad de la competencia que pudiera haber entre ellos, a tal punto que los trabajos de estos primeros diseñadores parecen distribuirse en diferentes inclinaciones estéticas y proyectos conceptuales de diseño diferentes que apuntan a diferentes tipos de potenciales clientes.

\section{BAF Week: El encuentro de productores, mediadores y público}

El diseño, para ser reconocido como valor, debe trascender las fronteras de la academia, la producción restringida, el círculo de pares, para llegar a un público amplio que pueda apropiarse simbólica y económicamente de sus productos.

$\mathrm{Si}$ es el conjunto de agentes y su sistema de relaciones el que hace a la valoración de productores, obras, productos y a la reproducción del universo de creencia, es necesario incluir también a aquellos que se apropian o consumen la creencia. En esa perspectiva se pueden recuperar algunos elementos que Bourdieu desarrolla sobre la dinámica de los campos y la conformación de los gustos, en relación con sus bases sociales, y donde particularmente discute la apropiación de los bienes de lujo, específicamente los bienes culturales (Bourdieu, 2000:223-253). Esto introduce la idea de un ajuste entre oferta y demanda como resultado de la sincronía objetiva de dos lógicas relativamente independientes: la de la producción y la del consumo.

La circulación y distribución de un bien o una práctica tiene como resultado disminuir su singularidad y su valor específico, amenazando la distinción de sus poseedores preexistentes. Los efectos benéficos de la distinción se desvanecerían con el tiempo si la producción de los bienes culturales (también regida por esa lógica dialéctica de la pretensión y distinción) no ofreciera permanentemente nuevos bienes o nuevas maneras de apropiarse de ellos. Esto introduce la idea de un ajuste entre oferta y demanda como resultado de la sincronía objetiva de dos lógicas relativamente independientes: la de la producción y la del consumo. Propone una homología entre los campos de producción especializada y los 
campos donde se determinan los gustos, donde, en medio de las luchas por la legitimidad y el cambio permanente, los productos encuentran su demanda. Para Bourdieu, el campo de producción no podría funcionar si no contara con unos gustos ya existentes. Eso es lo que permite realizarse al gusto de la demanda, ofreciéndole, en cada momento, el universo de bienes culturales como sistema de posibles estilísticos entre los cuales puede seleccionar el sistema de los rasgos constitutivos de su estilo de vida" (Bourdieu, 2000:228).

La homología funcional y estructural entre ambos sistemas reside en el hecho de que todos los campos especializados se organizan y estructuran según la misma lógica, según el capital específico acumulado, y en la coexistencia de posiciones dominantes y subordinadas, ortodoxia y herejía, antiguos y recién llegados, pretensión y distinción, etcétera (Bourdieu, 2000:230). Los gustos, en este esquema, dependen entonces del sistema de bienes ofrecidos, provocando en ese espacio un cambio en el sistema de gustos; mientras que, a la inversa, los cambios en los gustos, dados por transformaciones de las condiciones de existencia, conducen a transformaciones en el campo de producción, dando la ilusión de que hay cosas "para todos los gustos" o que "todo el mundo vende". Pero esa situación no es producto de una búsqueda intencionada, sino, más bien, el encuentro entre estos dos sistemas: producción y consumo.

Ese encuentro entre producción, mediación y consumo fue propiciado por un evento que surge en el año 2000 en el formato de "semana de la moda" conocido como BAF Week ${ }^{8}$. Podría decirse que la producción de los diseñadores que nos ocupan cobró visibilidad a partir de este evento que mostraba desfiles de colecciones nucleando las propuestas de los diseñadores-empresarios en un solo espacio. Esta semana de la moda constituyó uno de los principales aportes al desarrollo del diseño local, acercándolos a la prensa, clientes y público, reuniendo dos veces al año las colecciones de los diseñadores-profesionales que comenzaban a avanzar en sus carreras como empresarios, reforzando sus marcas individuales.

En esos inicios los diseñadores no contaban con gran visibilidad pública. Empezamos a llamar diseñadores que eran conocidos, pero no al mismo nivel que estamos manejando ahora. No tenían tanta difusión, prensa, tanta imagen, no tenían locales a la calle. (Entrevista Organización BAF Week, 2006). Al mismo tiempo, fue necesario reunir patrocinadores y auspiciantes para el evento, convencerlos del interés del proyecto y lograr apoyo tanto simbólico como económico. Se contactó al Director del Centro Metropolitano de Diseño, institución del Gobierno de la Ciudad de Buenos Aires que también iniciaba sus actividades en ese momento, y se intentó reunir a todos los sectores que podrían estar vinculados a este tipo de producción y pudieran aportar una cartera de diseñadores que dieran cuerpo e interés a la naciente semana de la moda.

Poco a poco fue consolidándose en términos de un escenario, de una arena colectiva. En ese sentido, puede pensarse la semana de la moda como un espacio donde se manifiesta el estado del espacio de producción, que se materializa. Al poner juntos los distintos actores en la especialidad de un evento cerrado y temporal, la semana de la moda deja visible en su puesta en escena características más amplias del campo: límites, posiciones, toma de posiciones y habitus (Entwistle y Rocamora, 2006:736).

En el caso local, sobre todo en el período de su conformación como espacio de producción legítimo, la semana de la moda aparece como un espacio más abierto que otras semanas de la moda más consolidadas, que representan los centros de la moda. Mientras que en otros 
casos el acceso se da a través de invitaciones personalizadas y bajo un estricto control, en el caso local el público en general puede acceder tanto a desfiles como a showrooms simplemente mediante la compra una entrada, de valor accesible, similar al costo de una entrada de cine.

Más allá de esa modalidad abierta y accesible, la semana de la moda representa un momento crítico en la vida cotidiana del campo como un todo y, más allá de mostrar las colecciones, tiene la función de producir, reproducir y legitimar el campo de producción y las posiciones de los agentes que juegan allí (Entwistle y Rocamora, 2006). A partir de esta idea es posible leer el significado que tiene la semana de la moda para los diseñadores y otros agentes, y su participación en la misma. Para los diseñadores introduce una temporalidad que organiza el trabajo anual, y también es un momento de prueba, de evaluación de su trabajo, el momento de ver "en qué está cada uno": Para mí el Fashion Buenos Aires es tener una fecha limite, es saber que tal día tengo que tener todo hecho, básicamente, plazos. (...) Es estar dentro del circuito, es estar en la palestra. Es donde vos ponés el cuerpo y te expones. (...) Fashion Buenos Aires, sabés que tiene que existir porque necesitamos un lugar donde mostrarnos (Entrevista Diseñadora, Empresaria, 2007).

Los propios diseñadores detectan elementos que tienen que ver con una puesta en común colectiva, con un capital simbólico que se potencia como aglutinador de las producciones individuales, a la vez que también emergen las limitaciones y problemas, particularmente en cuanto a lo que tiene que ver, por ejemplo, con las oportunidades de negocios que ofrece y también en la estructura misma. Yo no me puedo apoyar en el Fashion Buenos Aires. Está bien, es más poderoso porque justamente nos aglutina a todos y porque tiene un poder de negociación mayor pero no es mucho más sólido que cada uno de los proyectos individuales que se presentan ahí (Entrevista Diseñadora, Empresaria, 2006).

La BAF week logró seguir produciendo dos ediciones anuales reuniendo un importante caudal de público, junto con la producción de los diseñadores más destacados de esas primeras camadas de diseñadores-empresarios, en un promedio de 25 a 30 desfiles por edición y unos 45 showrooms. Los productores representados en desfiles y showrooms entre 2001 y 2005 permanecen relativamente constantes y formando parte casi de un mismo grupo. En 2006, el diario La Nación, se asocia con APSA y La Rural para seguir produciendo la semana de la moda BAF Week. Notablemente, a partir de esa edición, el evento comienza a incluir marcas "comerciales" junto al trabajo de los diseñadores que venía presentando y la cantidad de showrooms aumenta.

\section{“Hacerse un nombre”: La prensa y la movilización de capital simbólico}

Los críticos, en el esquema de Bourdieu -en el caso que nos ocupa, la prensa especializada-colaboran también en el trabajo de consagración que hace a la reputación tanto del periodista 'descubridor' que presenta algo novedoso a sus lectores, como del 'descubierto'. Su labor, si bien se envuelve en la ilusión de una garantía de juicio puramente estético, tiene efectos también en el valor económico de los productos, en la medida en que guían $\mathrm{y}$ orientan las tendencias entre compradores y vendedores. 
La prensa especializada contribuye a entender cómo se dieron los procesos de mediación respecto de un público amplio. Cumpliendo una suerte de rol pedagógico ha construido un pasado, explicando de qué se trata el diseño, difundiendo el concepto de "diseño de autor", identificando sus espacios de expresión principales, a la vez que genera lazos con otras zonas de acción como el campo artístico que trasladando su capital simbólico ejercen una acción legitimadora.

En el proceso de consolidación del diseño de indumentaria local la prensa explora y amplifica las relaciones que se han dado con el campo artístico. Esto tiene que ver con dos ejes: uno que construye la historia del propio espacio de producción del diseño desde la crítica especializada, y otro que tiene que ver con instituciones artísticas que han incluido el diseño en sus muestras y que han funcionado como espacios de legitimación y prestigio para los diseñadores.

Partiendo de la lectura de la prensa especializada en moda, es posible plantear cómo se construye cierta definición de un concepto y se difunde masivamente a propósito del diseño, particularmente el de indumentaria: el “diseño de autor”. Esta categoría refleja la forma de pensar en un estilo específico de diseño, que circula en ciertos espacios, primero de manera más restringida, luego amplificándose en el uso (Douglas, 1998). Asociada a la indumentaria, comenzó a circular justamente para dar cuenta de un emergente novedoso en la producción y nominar un conjunto de producciones en indumentaria, textiles y accesorios que no podían ser atribuidas a las marcas locales ya instaladas o a los modistos más tradicionales y que mayormente se asocian a emprendimientos centrados en la producción de diseñadores formados en la universidad, particularmente la Universidad de Buenos Aires ${ }^{9}$ (Miguel, 2019). Otros trabajos analíticos resaltan la perspectiva que nutre un relato que vincula el "diseño" con el momento de efervescencia en la producción estética y artística característica de la vuelta a la democracia en los años 80 , la creación de la carrera de Diseño de Indumentaria y Textil en la Universidad de Buenos Aires, y la crisis de 2001 como el momento en que esas producciones cobran visibilidad (Saulquin 2006, Joly 2012, Tuozzo y López, 2013). A partir de las interpretaciones de periodistas de moda y especialistas vinculados al diseño profesional, se agrupó bajo este concepto a los diseñadores profesionales que se pusieron al frente de sus emprendimientos, el estilo de diseño que producían y los espacios donde se los podía encontrar.

A medida que se gana visibilidad, reconocimiento y legitimación, también se van definiendo posiciones y jerarquías que la prensa contribuye a sedimentar. Entre los "conocedores" existe un conocimiento tácito compartido sobre la valoración de las diferentes manifestaciones de diseño que coexisten tal vez en un mismo espacio (por ejemplo, en el barrio de Palermo) pero que no "pesan" lo mismo. Las ferias y "las marcas comerciales" pueden pretender el atributo de diseño, pero son algo distinto. La oferta de estos espacios no es reconocida con la legitimidad de aquella de los diseñadores que tienen su "firma" y locales propios.

Por otro lado, tenés la oferta de diseño de autor de ferias de cualquiera que se pone a bordar botones (Entrevista a Diseñadora-Empresaria, 2005).

El problema son las marcas soberbias, que en vez de aportar una mirada interesante destruyen el estilo auténtico del barrio. [Palermo] Creció, fue una ex- 
plosión de creatividad, pero al mismo tiempo se contaminó con formatos copiados, con mucha inversión y pocas ideas. (Diseñadoras-empresarias, 2015)

Para ilustrar la forma en que la prensa opera dando lugar en sus notas a los diseñadores, es posible cuantificar la cantidad de resultados de notas que mencionan a cada uno dentro del grupo La Nación (diario, suplementos, revista Ohlalá, etc.), publicación seleccionada ya que es la que le dedica mayor cantidad de notas a estos temas y, además, entre los años 2006 y 2014, fue uno de los principales organizadores de la semana de la moda BAF Week en el Cuadro 1. Los nombres de diseñadores fueron seleccionados a partir de los listados que aparecen en catálogos y libros de especialistas que coinciden en agrupar a estos diseñadores como referentes consagrados del diseño local y como "autores" (Lescano 2004, Saulquin 2006 y 2011, Acevedo Díaz 2011, Chiesa et al. 2012, Acosta et al. 2013).

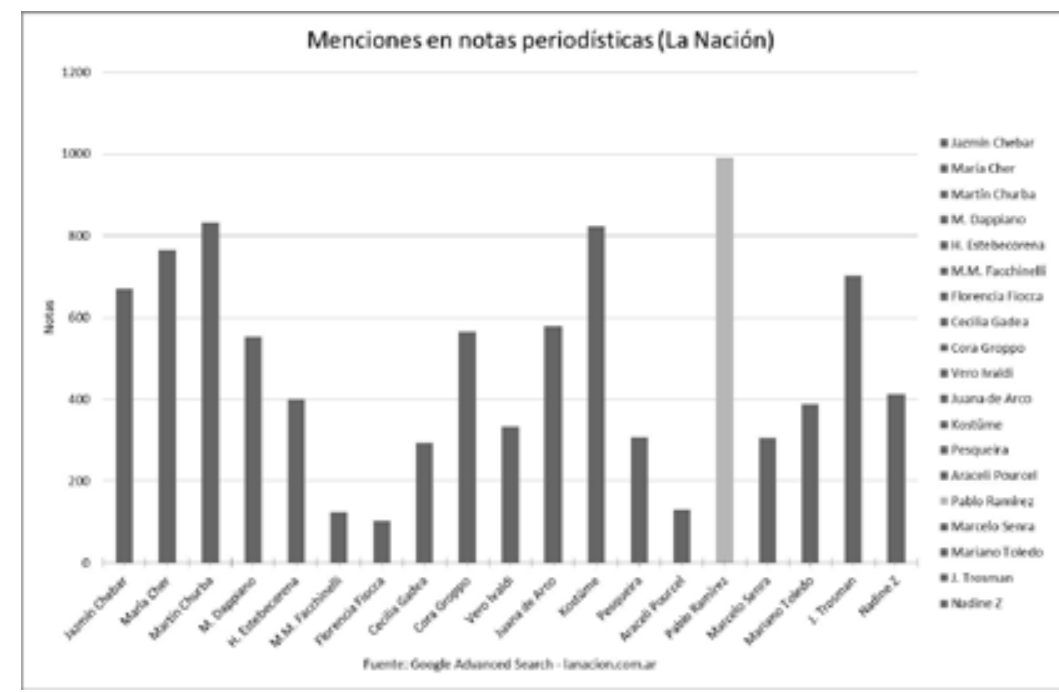

Cuadro 1. Menciones de diseñadores en notas periodísticas - La Nación.

Aquí puede observarse que aquellos con más presencia en las notas de prensa no necesariamente son aquellos con negocios más exitosos (en ventas, por ejemplo), sino que siguiendo el esquema de producción de la creencia artística que propone Bourdieu, parece regir incluso una idea de rechazo de lo comercial. En el campo amplio de la producción cultural, el capital útil es el capital simbólico: aquel que otorga prestigio o autoridad, aquel que, al "hacer un nombre", se convierte en "capital de consagración" que confiere la facultad de ubicar en una posición de privilegio objetos o personas (Bourdieu, 1995:224). Esto quiere decir que la forma que el capital económico tiene de asegurarle a su poseedor los beneficios específicos de este campo, es reconvirtiéndose en capital simbólico; es decir: adecuando la lógica económica a la lógica específica del espacio de producción específico. 


\section{Comentarios finales}

Este esquema interpretativo permite trabajar analíticamente en un plano relacional sobre producción y circulación de bienes culturales, poniendo el foco tanto en los productores, como en las instancias de intermediación, intermediarios y un público cercano, en cierta medida, fiel a la creencia. Así, recuperamos para estos espacios de producción la idea de que allí es eficaz una ideología carismática que origina la cuestión del productor aparente, del creador increado: la figura del genio, del autor iluminado, del creador y la creencia en esos roles. En este sentido,

El productor del valor de la obra de arte no es el artista, sino el campo de producción como universo de creencia que produce el valor de la obra de arte como fetiche al producir la creencia en el poder creador del artista (Bourdieu, 1995:339).

Esto apunta a esclarecer que la obra, la producción en estos espacios en general, es, en última instancia, un producto colectivo y social a la vez que individual. Sin la creencia de que las obras son obras, no sería posible hablar de las mismas. La "producción de la creencia" es una empresa colectiva, que requiere la movilización de energía y recursos compartidos, en distintas proporciones, por el conjunto de agentes implicados en el campo. El valor de la obra dado por la creencia surge, en última instancia, del conjunto de las prácticas que se dan entre productores, consumidores y quienes hacen a la mediación entre ellos de diversas maneras.

Es decir que la obra de arte (o el atributo estético/simbólico plasmado en un producto determinado) sólo es un objeto simbólico provisto de valor si es conocido y reconocido como tal, o, en otras palabras, si está instituido socialmente por un conjunto de productores y espectadores que detentan las competencias necesarias para conocerlo y reconocerlo en esos términos. Bajo esta perspectiva, comprender este tipo de producciones implica "no sólo la producción material de la obra, sino también la producción del valor de la obra o, lo que viene a ser lo mismo, de la creencia en el valor de la obra" (Bourdieu 1995:339) y, no menos importante, la creencia en el valor de sus productores, y de aquellos que pueden ser capaces de apreciar "genuinamente" el valor de esa obra. En ese sentido, a la par que se desarrolla y se complejiza el espacio productivo, la dinámica colectiva genera un conocimiento tácito sobre lo que es diseño, que es construido jerárquicamente como concepto polisémico, donde algunas definiciones sobre productores y productos, "valen" más que otras. Conocimiento que trasciende las fronteras del mercado de la moda y que es preciso desentramar en el análisis.

Esto resalta conceptualizaciones que resultan aún hoy productivas para entender algunas de las dinámicas que interesa analizar, en términos de delimitar un espacio de producción simbólica particular. Plantear una serie de posiciones, roles y relaciones en espacios donde, pese a tratarse de espacios de producción comerciales, muchas veces persiste, la lógica opaca del "talento", del "creador". Donde sigue estando presente la idea de que la producción más conceptual (restringida) ocupa una posición dominante, atrae mayor atención, parece producir los "talentos" más importantes y construye discursiva y simbólicamente su propia práctica con un mayor grado de confianza. Estos aportes pueden resultar úti- 
les para desentramar la forma en que esas ideas se reproducen desde su productividad y positividad y los fundamentos sociales en que se sostienen, que son en última instancia relaciones de poder.

\section{Notas}

1. Versiones preliminares de este trabajo fueron presentadas en las VIII Jornadas de Etnografía y Métodos Cualitativos, Buenos Aires, Instituto de Desarrollo Económico y Social, 2016 y en el III Coloquio de Investigación y Desarrollo en Diseño, Buenos Aires, Universidad de Palermo, 2018. Agradezco los comentarios allí recibidos.

2. La idea de campo (Bourdieu, 1988, 1990, 1991, 1995), supone entre dichos principios estructurantes, por un lado, la existencia de un capital específico en juego y actores dotados de habitus específicos. Por otro, implica la existencia de intereses comunes, generales, asociados al hecho de participar en el juego, vinculados a la existencia misma del campo (Bourdieu, 1990:135-140). Esto da origen a una complicidad entre los agentes que subyace y trasciende todo antagonismo, que producen creencia en el valor de lo que está en juego y no cuestionan los fundamentos del campo específico, reproduciendo así las bases de su funcionamiento.

3. El trabajo de campo se realizó en el marco de los proyectos UBACYT 20020110200278BA y $20020130200279 B A$, acreditados y financiados por la Universidad de Buenos Aires.

4. Los diarios Clarín, La Nación, Página/12 publican contenidos que permiten condensar enunciados e interpretar distintos elementos en relación con la moda en un público más amplio que el de las revistas de moda, aunque muestren producciones más simples que las revistas sobre moda.

5. Detalles a propósito de la formación académica local, centrada en el estudio del diseño como área proyectual, pueden encontrarse en Bonsiepe (1999), Fernández y Bonsiepe (2008), Devalle (2009).

6. Con la salida de la llamada Convertibilidad del Peso, en Argentina, particularmente en la ciudad de Buenos Aires, se reactiva el sector del textil y la confección. El diseño aplicado a la producción de indumentaria y textiles experimentó un crecimiento destacado en la ciudad de Buenos Aires. Entre 2004 y 2010 la actividad de confección de prendas de vestir, considerada industria de diseño intensiva, experimentó una variación del 176.26\% (Observatorio de Industrias Creativas 2012:88).

7. Proyecto gestado por diseñadores que pretendía ofrecer -en la Galería Larreta, ubicada en Florida y Santa Fe, en el barrio de Retiro- un espacio y un circuito cultural, con una característica fundamental: el cuidado de la oferta de diseño tanto conceptualmente como en los espacios comunes y particulares.

8. La primera edición albergó 21 desfiles y 37 showrooms. La cantidad de visitantes se calculó en unas 35 mil personas. Una cifra significativa si se piensa en la incipiente difusión que tenían los diseñadores y sus productos.

9. Un exponente muy dinámico del diseño profesional se vincula con la carrera de Diseño de Indumentaria y Textil (DIT) en la Facultad de Arquitectura y Urbanismo de la Universidad de Buenos Aires. Esta carrera se creó en los años 1988-1989 y representó un paso importante hacia la institucionalización y profesionalización del diseño en esas áreas de produc- 
ción en Argentina. Ha sido y continúa siendo de las más numerosas del país en matrícula de estudiantes y logró posicionarse como referente en la región, ganando un reconocimiento internacional gracias a los trabajos y logros de sus estudiantes, egresados y docentes.

\section{Bibliografía}

Acevedo Díaz, C. (2011). Moda. Nuevo diseño argentino. Buenos Aires: Olmo Ediciones. Acosta, A. et al. (2013). INTI mapa de diseño 101 diseñadores de autor. San Martín: Instituto Nacional de Tecnología Industrial.

Bonsiepe, G. (1999) Del objeto a la interfase: mutaciones del diseño, Buenos Aires: Infinito. Bourdieu, P. (1988). Cosas Dichas. Buenos Aires: Gedisa. . (1990). Alta costura y alta cultura. En: Sociología y cultura. México: Grijalbo. . (1991). El sentido práctico. Madrid: Taurus. . (1995). Las reglas del arte: génesis y estructura del campo literario. Barcelona: Anagrama. . (2000). La distinción: criterios y bases sociales del gusto. Madrid: Taurus. . (2003). Creencia artística y bienes simbólicos: elementos para una sociología de la cultura. Buenos Aires: Aurelia Rivera.

Bourdieu, P. \& Delsaut, Y. 1975. "Le couturier et sa griffe : contribution à une théorie de la magie." Actes de la recherche en sciences sociales 1:7-36.

Callon, M.; Meadel, C. \& Rabeharisoa, V. (2005). The Economy of Qualities. In A. Barry, D. Slater (eds.) The Technological Economy. London: Routledge.

Chiesa, M. et al. (2012). Buenos Aires es tendencia: diseño de indumentaria en la era digital. Buenos Aires: Sudamericana.

Devalle, V. (2009). La travesía de la forma. Emergencia y consolidación del Diseño Gráfico (1948-1984). Buenos Aires: Paidós.

Dimaggio, P. (1987). Classification in art. American Sociological Review, 52, (4), 440-455.

Douglas, M. (1998). Estilos de pensar. Ensayos críticos sobre el buen gusto. Barcelona: Gedisa.

Entwistle, J. y Rocamora, A. (2006). “The field of fashion materialized: a study of London Fashion Week. Sociology 40 (4), 735-751.

Fernández, S. y Bonsiepe, G. (Comp.) (2008). Historia del diseño en América Latina y el Caribe. Sao Paulo: Editora Blücher.

Guerschman, B. y Vargas, P. (2007). Quilombo y apuesta: apuntes etnográficos sobre la crisis argentina de 2001 a través de la mirada del mundo del diseño. Avá, (11), 39-62.

Joly, V. (2012). Arte, moda y consumo cultural. Cuadernos del Centro de Estudios en Diseño y Comunicación. Ensayos, (39), 283-294.

Lescano, V. (2004). Followers of fashion: falso diccionario de la moda. Buenos Aires: Interzona. Marino, P. et al. (2010). Diseño de indumentaria de autor en Argentina. Buenos Aires: INTI. Miguel, P. (2013). Emprendedores del diseño. Aportes para una sociología de la moda. Buenos Aires: Eudeba.

. (2015) La estetización del espacio y la espacialización de lo estético en Buenos Aires. Una mirada desde la producción. Bifurcaciones. Revista de estudios culturales urbanos, (20). Disponible en: www.bifurcaciones.cl 
. (2019). Más allá del autor. La construcción pública del diseño de indumentaria en Argentina. Cuadernos del Centro de Estudios de Diseño y Comunicación, (76):161-177. McRobbie, A. (1998). British fashion design. Rag trade or image industry. Londres: Routledge. Observatorio de Industrias Creativas (2012). Anuario de Industrias Creativas Ciudad de Buenos Aires 2011. Buenos Aires: Ministerio de Desarrollo Económico, GCBA.

Pérez Zelaschi, M. (2012). Observatorio de Tendencias. Cuadernos del Centro de Estudios en Diseño y Comunicación. Ensayos, (42), 35-42.

Rocamora, A. (2006). Paris Capitale de la Mode: Representing Fashion in the Media. In: Fashion's World Cities. Berg Publishers, pp. 43-54.

Rocamora, A. (2015). High Fashion and Pop Fashion: The Symbolic Production of Fashion in Le Monde and The Guardian. Fashion Theory (5),2: 123-142.

Saulquin, S. (2006). Historia de la moda argentina: Del miriñaque al diseño de autor. Buenos Aires: Emecé.

Tuozzo, M. V.y López, P. (2013). Moda y Arte. Campos en intersección. Cuadernos del Centro de Estudios en Diseño y Comunicación. Ensayos, (44), 123-134.

Vargas, P. (2013). Diseñadores y emprendedores. Una etnografía sobre la producción y el consumo de diseño en Buenos Aires. La Plata: Al Margen.

\begin{abstract}
This paper analyzes the process of valorization, legitimacy and increasing autonomy acquired by clothing design since 2000 in Buenos Aires, focusing on professional designers-entrepreneurs. Using as trigger Pierre Bourdieu's concern for the worlds of "high culture", the cultural, aesthetic and symbolic production, and his ideas about "univers of belief" to articulate the presence of different actors with particular logics in a relational space shared by producers, products, intermediaries and the public, where tastes and preferences are built, reproduced and renewed, where positions are negotiated and hierarchies are raised.
\end{abstract}

Keywords: Design - Clothing - Symbolic production - Entrepreneurism.

Resumo: Este trabalho analisa o processo de valorização, legitimação e crescente autonomia adquirida pelo design de vestuário do ano 2000 em Buenos Aires, enfocando designers profissionais-empreendedores. Utilizo a preocupação pelos mundos da "alta cultura", a produção de valores culturais, estéticos e simbólicos na obra de Pierre Bourdieu, suas idéias sobre os "universos da crença" para articular a presença de diferentes atores com lógicas particulares em um espaço relacional compartilhado por produtores, produtos, intermediários e público, onde os gostos e preferências são construídos, reproduzidos e renovados, onde posições são negociadas e onde produzem-se as hierarquias.

Palavras chave: Design - Vestuário - Produção simbólica - Empreendedorismo.

[Las traducciones de los abstracts fueron supervisadas por el autor de cada artículo] 